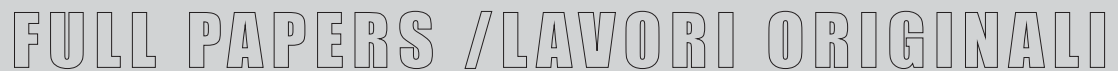

\section{Sinergismo in vitro tra rokitamicina e cotrimossazolo in S. pyogenes e S. pneumoniae}

\author{
Simona Roveta', Anna Marchese', Eugenio A. Debbia', Roberto Bandettini² \\ 'Università di Genova, DISCAT, Sezione di Microbiologia, Genova \\ ${ }^{2}$ Istituto G. Gaslini, Genova
}

Key words: antibiotic synergism, streptococci, macrolides

In vitro synergism between rokitamycin and cotrimoxazole against S. pyogenes and S. pneumoniae

\section{SUMMARY}

Background: Synergism between cotrimoxazole (SXT) and rokitamycin (ROK) was observed carrying out antimicrobial susceptibility tests on S. pyogenes and S. pneumoniae with the disk diffusion method (CLSI, 2005). The aim of this study was to confirm this phenomenon on a large number of isolates displaying different macrolide resistance phenotypes.

Methods: Synergism between SXT and ROK on 104 S. pyogenes and 102 S. pneumoniae recently isolated was detected by a double-disk screening test. Time kill experiments were also performed on representative strains adopting standard procedures (CLSI 2005).

Results: The combination of SXT plus ROK was synergistic against $93 \%$ S. pyogenes strains and 5 I\% S. pneumoniae strains. On pneumococci SXT-S this percentage arise to $64 \%$, while, on SXT-R it was $29 \%$. In no instances antagonism was demonstrated. Synergism was not observed against $S$. pyogenes strains showing $\mathrm{cMLS}_{\mathrm{B}}$ phenotype. In S. pneumoniae no relationship between different mechanisms of macrolide resistance and the results of interactions was found. Results of time-kill experiments confirmed those obtained with double-disk assay in all the strains tested.

Conclusion: Synergism between SXT and ROK was more frequently encountered among S. pyogenes than S. pneumoniae strains. This drug association may be synergistic or not when acting on different strains of the same bacterial species. Different macrolide-resistance mechanisms (reduced binding due to modification of the $50 \mathrm{~S}$ subunit or efflux pump) among the various bacteria may eplain the observed differences.

\section{INTRODUZIONE}

I macrolidi esplicano la loro attività sulla cellula batterica interagendo con la subunità ribosomale 50S (si legano al 23S RNA e impediscono l'assemblaggio dell'unità), bloccando quindi la sintesi proteica. La resistenza a questa classe di antibiotici può essere conferita da una modificazione enzimatica del ribosoma batterico che avviene ad opera di una metilasi che catalizza un processo di metilazione nel 23S rRNA. I geni erm codificano enzimi di questo tipo e la resistenza da essi mediata può essere inducibile (iMLS $\mathrm{B}_{\mathrm{B}}$ o costitutiva $\left(\mathrm{cMLS}_{\mathrm{B}}\right)$. La refrattarietà ai macrolidi negli streptococchi è frequentemente dovuta (a causa delle presenza del gene mef) a un meccanismo di efflusso che conferisce resistenza alle molecole a 14 e 15 atomi ma non a macrolidi a 16 atomi, lincosamidi o streptogramine (fenotipo $M)(5,7)$.

L'associazione di 5 parti di sulfametossazolo con 1 parte di trimethoprim viene denominata cotrimossazolo: la combinazione di questi due farmaci agisce bloccando due passaggi distinti del metabolismo degli acidi folici. Il sulfametossazolo, analogo dell'acido para-aminobenzoico, inibi- sce competitivamente la sintesi batterica dell'acido diidrofolico agendo a livello dell'enzima diidropteroato-sintetasi (DPS).

Il trimetoprim, legandosi all'enzima diidrofolatoreduttasi (DHFR) batterica ne inibisce l'azione, prevenendo, in tal modo, la sintesi dell'acido tetraidrofolico (essenziale per la sintesi degli acidi nucleici) dall'acido diidrofolico.

La resistenza al trimethoprim-sulfametossazolo si realizza mediante la sintesi di enzimi DPS o DHFR (dovuta a determinanti genetici situati su plasmidi o trasposoni) che presentano un'affinità molto minore per l'inibitore rispetto a quelli omologhi cromosomici: si instaura così una via metabolica alternativa che consente di aggirare l'azione del farmaco antibatterico.

Un meccanismo alternativo di resistenza, ma che incide in maniera molto minore, è l'iperproduzione di acido paraminobenzoico oppure di DHFR (rispettivamente per la resistenza al sulfametossazolo e al trimethoprim) (10).

In alcuni antibiogrammi relativi a ceppi di $S$. pyogenes e $S$. pneumoniae (eseguiti con la metodica classica Kirby-Bauer) (3) sono stati osservati 
occasionalmente degli aloni di inibizione riconducibili ad un possibile effetto di interazione sinergica tra cotrimossazolo (SXT) e macrolidi a 16 atomi (figura I).

In seguito a questo riscontro è stato eseguito uno screening su un ampio numero di streptococchi e, per approfondire ulteriormente l'indagine, sono state eseguite delle curve di batteriocidia (timekill) su alcuni ceppi rappresentativi dei differenti fenotipi di resistenza ai macrolidi.

\section{MATERIALI E METODI}

Sono stati analizzati 104 ceppi di $S$. pyogenes e 102 ceppi di $S$. pneumoniae di recente isolamento (2005-2006) provenienti dalla collezione della Sezione di Microbiologia del DISCAT (Università degli Studi di Genova) e dall'Ospedale Pediatrico "Istituto G. Gaslini" di Genova.

Ciascun stipite resistente all'eritromicina è stato ben caratterizzato per il fenotipo di resistenza ai macrolidi (cMLSB, iMLSB oppure M) effettuando il test di induzione (9): sulla superficie di una piastra di terreno Mueller-Hinton (MH) agar (addizionato con sangue di montone $0.5 \%$ ) insemenzata con una sospensione di torbidità 0.5 McFarland del ceppo da esaminare sono stati posti 2 dischetti di eritromicina $(15 \mu \mathrm{g})$ e clindamicina $(2 \mu \mathrm{g})$ a una distanza di $1.5-2 \mathrm{~cm}$. Dopo 18 ore di incubazione a $37^{\circ} \mathrm{C}$, la comparsa di inibizione attorno al dischetto di clindamicina (diametro $>19 \mathrm{~mm}$ ) indicava il fenotipo $\mathrm{M}$, mentre la deformazione dell'alone di inibizione attorno al dischetto di clindamicina nella zona adiacente a quello di eritromicina indicava il fenotipo iMLSB. L'assenza di un alone di inibizione attorno ad entrambi i dischetti indicava invece resistenza costitutiva $\left(\mathrm{cMLS}_{\mathrm{B}}\right)$.

Lo screening per evidenziare l'eventuale sinergismo cotrimossazolo-macrolide a 16 atomi è stato eseguito con la metodica del doppio dischetto ponendo il dischetto di SXT a $2 \mathrm{~cm}$ da quello di rokitamicina (ROK).

Alcuni stipiti, rappresentativi di ciascun fenotipo di resistenza ai macrolidi ( cMLS $_{\text {в }}$, iMLS $_{\text {в }}$ ed M) e selezionati in modo tale da avere tutte le combinazioni possibili delle resistenze cotrimossazolomacrolidi sono stati in seguito ulteriormente studiati con l'esecuzione di curve di batteriocidia (time-kill) per verificare l'interazione positiva dell'associazione tra le molecole. Le curve di batteriocidia sono state eseguite con la metodica classica (6).

La coltura in fase di crescita esponenziale è stata diluita in brodo fresco Mueller-Hinton (MH) addizionato con $5 \%$ di sangue lisato sino ad una concentrazione compresa tra $10^{6}$ e $10^{7} \mathrm{CFU} / \mathrm{ml}$, si sono quindi allestiti 4 campioni: uno di controllo, il secondo con SXT alla concentrazione di 1/2 MIC, il terzo con il macrolide alla stessa dose ed infine uno contenente l'associazione di farmaci ad 1/2 MIC ciascuno. Dopo un prelievo al tempo 0 (prima di addizionare l'antibiotico) per stimare la carica batterica iniziale, $i$ campioni sono stati incubati a una temperatura di $37^{\circ} \mathrm{C}$ in $\mathrm{CO}_{2}$, allo scadere delle 2, 6 e 24 ore ( 8 in caso di $S$. pneumoniae), è stata eseguita la conta dei sopravvissuti.

\section{RISULTATI}

Lo screening ha rivelato un'interazione positiva tra cotrimossazolo e rokitamicina sul $93 \%$ dei ceppi di $S$. pyogenes saggiati (tabella 1); in particolare, tra questi il $60.6 \%$ era sensibile ai macrolidi, il $16.3 \%$ presentava fenotipo $\mathrm{M}$ e 1' $8.7 \%$ il fenotipo iMLS non è stata evidenziata interazione presentavano fenotipo $\mathrm{cMLS}_{\mathrm{B}}$.

Per quanto riguarda $S$. pneumoniae, invece, la percentuale di interazione positiva riscontrata durante lo screening è stata del 51\%. Negli pneumococchi SXT-S questa percentuale saliva al $64 \%$, mentre in quelli SXT-R era solamente del $29 \%$. Nei ceppi in cui non è stato evidenziato sinergismo il fenotipo di resistenza ai macrolidi non era sostanzialmente differente da quelli in cui l'interazione è risultata positiva (tabella 2).

Il saggio dell'attività antibatterica dell'associazione cotrimossazolo-rokitamicina tramite time-kill su alcuni ceppi rappresentativi con differenti fenotipi di sensibilità ai due antibiotici ha riconfermato i risultati ottenuti con lo screening del doppio disco. Su tutti i ceppi in cui era stato riscontrato sinergismo è stata osservata una diminuzione del 99\% delle $\mathrm{CFU} / \mathrm{ml}$ rispetto agli antibiotici da soli. La mancanza di effetto sinergico è stata osservata solo sui ceppi (fenotipo $M L S_{B}$ ) che avevano già manifestato questa caratteristica durante lo screening preliminare (figure II-V).

\section{CONCLUSIONI}

Il sinergismo SXT-macrolide a 16 atomi osservato in piastra tramite gli aloni di inibizione dei dischetti di antibiotico sembra essere più significativo per i ceppi di $S$. pyogenes piuttosto che per i ceppi di $S$. pneumoniae. In $S$. pyogenes l'interazione positiva appare più influenzata dalla sensibilità dello stipite ai macrolidi che a SXT. In S. pneumoniae, invece, il sinergismo sembra essere indipendente dalla sensibilità del ceppo ai macrolidi, mentre appare più influenzato dalla sensibilità a SXT. 
L'associazione di farmaci ha prodotto sui vari microrganismi analizzati effetti differenti anche nell'ambito della stessa specie e su stipiti con stesso fenotipo di resistenza ai macrolidi: questi risultati eterogenei potrebbero essere imputati alla varietà dei meccanismi di resistenza a queste molecole.

Come è noto, infatti, la resistenza ai macrolidi può manifestarsi nei batteri attraverso fenotipi differenti che rispecchiano un diverso genotipo; i due più comuni sono l'alterazione della subunità ribosomale $50 \mathrm{~S}$ (con conseguente ridotta affinità nei confronti del farmaco) oppure l'efflusso attivo dell' antibiotico dalla cellula $(1,4)$. L'inattivazione diretta dell'antibiotico è un meccanismo molto meno comune riscontrato ad es. nelle Enterobacteriaceae ad opera di esterasi (2).

I determinanti genetici della resistenza ai macrolidi si trovano in plasmidi e trasposoni (alcuni ceppi ne possono albergare anche più di un tipo), spesso associati con geni veicolanti altre resistenze antibiotiche. La presenza dei geni erm conferisce sempre resistenza ad alto livello a tutti i macrolidi a 14 e 15 atomi e, se la metilasi è prodotta costitutivamente, si può avere anche resistenza alle molecole a 16 atomi, lincosamidi, streptogramine e ketolidi.

Il livello di resistenza conferito è inoltre correlato con il grado di metilazione: alcuni enzimi catalizzano solo la monometilazione, altri la dimetilazione, altri ancora $(\operatorname{ErmB})$ catalizzano sia la mono- che la dimetilazione. In $S$. pyogenes i livelli di dimetilazione sono maggiori che in $S$. pneumoniae $(5,7)$.

Alcuni studi condotti su isolati clinici di S. pneumoniae contenenti geni erm hanno dimostrato che i livelli basali di metilazione ribosomale variano da un ceppo all'altro $(8,11)$. In questo studio è stato riscontrato che in presenza di fenotipo $\mathrm{MLS}_{\mathrm{B}}$ è possibile osservare sinergismo SXTmacrolide a 16 atomi soltanto in alcuni ceppi di S. pneumoniae: negli stipiti in cui i livelli basali di metilazione sono minori vi può essere un superamento della resistenza al macrolide grazie al cotrimossazolo.

Il meccanismo con cui tale fenomeno avviene può essere spiegato tramite l'inibizione della metil-transferasi da parte del cotrimossazolo (7), in quanto la metilazione che media la resistenza richiede s-adenosil-metionina come donatore del gruppo metilico (5).

Per quanto riguarda invece gli stipiti con fenotipo di resistenza ai macrolidi di tipo $\mathrm{M}$ in cui si è osservato sinergismo SXT-macrolide a 16 atomi, si può ipotizzare che, in questo caso, il macrolide consenta il superamento della resi- stenza al cotrimossazolo bloccando la via metabolica alternativa in seguito al blocco generalizzato della sintesi proteica. Anche in questo caso la varietà dei risultati osservati può essere giustificata dal fatto che più di un enzima (DPS o DHFR) può essere coinvolto nel meccanismo di resistenza al cotrimossazolo (10).

Per giustificare gli eterogenei risultati riscontrati possono essere avanzate anche altre ipotesi: differenze tra i ribosomi (o una diversa modalità di legame dei macrolidi al bersaglio) potrebbero essere sufficienti a spiegare il differente comportamento osservato.

Anche differenze tra i vari microrganismi nell'accumulo intracellulare delle molecole, piuttosto che differenze nella struttura ribosomica, potrebbero fornire una spiegazione delle differenze osservate.

Tabella I. Interazione tra cotrimossazolo e macrolidi a 16 atomi in S. pyogenes

\begin{tabular}{|c|c|c|c|}
\hline \multirow{2}{*}{$\begin{array}{c}\text { SXT } \\
\text { (n. ceppi e \%) }\end{array}$} & \multirow{2}{*}{$\begin{array}{l}\text { Fenotipo di } \\
\text { resistenza } \\
\text { ai macrolidi }\end{array}$} & Sinergismo & Indifferenza \\
\hline & & \multicolumn{2}{|c|}{ (n. ceppi e \%) } \\
\hline \multirow[t]{5}{*}{ S $79(76 \%)$} & $\mathrm{S}$ & $48(60.8 \%)$ & 0 \\
\hline & $M$ & 13 (16.4\%) & 0 \\
\hline & iMLS & $8(10.1 \%)$ & 0 \\
\hline & CMLS & $6(7.6 \%)$ & 4 \\
\hline & TOT & 75 (94.9\%) & $4(5.1 \%)$ \\
\hline \multirow[t]{5}{*}{ R 25 (24\%) } & $\mathrm{S}$ & $15(60 \%)$ & 0 \\
\hline & M & $4(16 \%)$ & 0 \\
\hline & iMLS & I (4\%) & 0 \\
\hline & $c M L S_{B}$ & $2(8 \%)$ & $3(12 \%)$ \\
\hline & TOT & $22(88 \%)$ & $3(12 \%)$ \\
\hline TOTALI I04 & $\mathrm{S}$ & 63 (60.6\%) & 0 \\
\hline \multirow[t]{4}{*}{$(100 \%)$} & $M$ & 17 (16.3\%) & 0 \\
\hline & iMLSB & $9(8.7 \%)$ & 0 \\
\hline & $c M L S_{B}$ & 8 (7.7 \%) & 7 (6.7\%) \\
\hline & TOT & 97 (93.3\%) & $7(6.7 \%)$ \\
\hline
\end{tabular}

Tabella 2. Interazione tra cotrimossazolo e macrolidi a 16 atomi in S. pneumoniae

\begin{tabular}{|c|c|c|c|}
\hline \multirow{2}{*}{$\begin{array}{c}\text { SXT } \\
\text { (n. ceppi e \%) }\end{array}$} & \multirow{2}{*}{$\begin{array}{l}\text { Fenotipo di } \\
\text { resistenza } \\
\text { ai macrolidi }\end{array}$} & Sinergismo & Indifferenza \\
\hline & & \multicolumn{2}{|c|}{ (n. ceppi e \%) } \\
\hline \multirow[t]{4}{*}{ S $64(62.7 \%)$} & $\mathrm{S}$ & $26(40.6 \%)$ & $15(23.5 \%)$ \\
\hline & $M$ & $8(12.5 \%)$ & $3(4.7 \%)$ \\
\hline & cMLS & $7(10.9 \%)$ & $5(7.8 \%)$ \\
\hline & TOT & $41(64 \%)$ & $23(36 \%)$ \\
\hline \multirow[t]{4}{*}{ R 38 (37.3\%) } & $S$ & 5 (I3.2\%) & $15(39.5 \%)$ \\
\hline & $M$ & $3(7.9 \%)$ & $4(10.5 \%)$ \\
\hline & сMLS & $3(7.9 \%)$ & $8(21 \%)$ \\
\hline & TOT & I I (29\%) & $27(7 \mid \%)$ \\
\hline TOTALI I02 & $\mathrm{S}$ & $31(30.4 \%)$ & $30(29.4 \%)$ \\
\hline \multirow[t]{3}{*}{$(100 \%)$} & $M$ & II (10.8\%) & $7(6.9 \%)$ \\
\hline & ¡MLS & $10(9.8 \%)$ & $13(12.7 \%)$ \\
\hline & TOT & $52(5 \mid \%)$ & $50(49 \%)$ \\
\hline
\end{tabular}




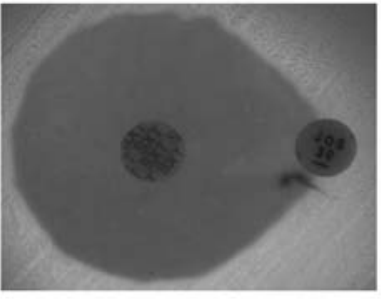

A

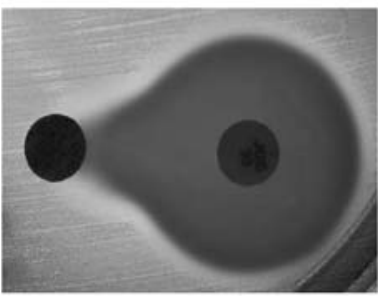

B

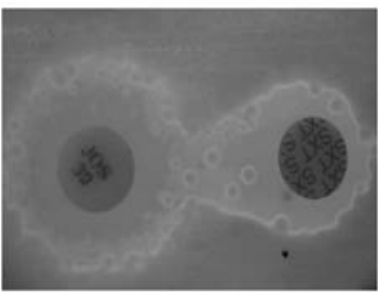

C

Figura I. Interazione tra SXT e macrolide a 16 atomi in un ceppo sensibile al cotrimossazolo e resistente ai macrolidi (A), un ceppo resistente al cotrimossazolo e sensibile ai macrolidi (B), un ceppo resistente a cotrimossazolo e macrolidi (C)

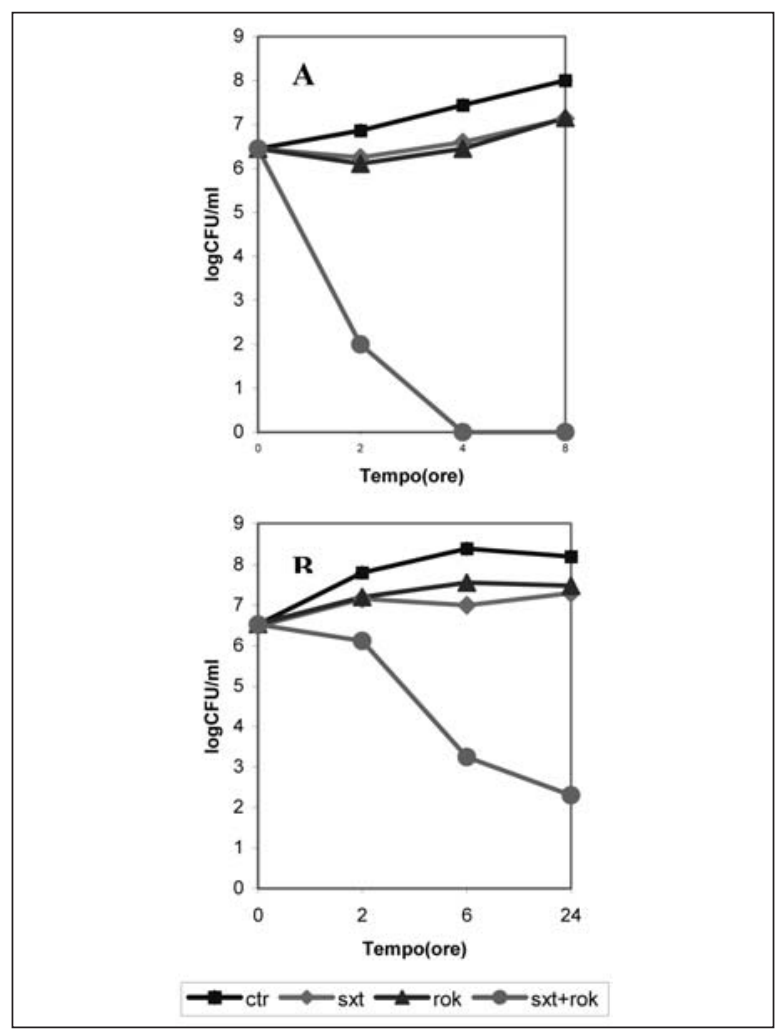

Figura II. Curve di battericidia relative a un ceppo di S. pneumoniae (GEI8) sensibile sia a SXT che ai macrolidi (A) e un ceppo di S. pyogenes (I3/2) resistente a SXT e sensibile ai macrolidi (B)
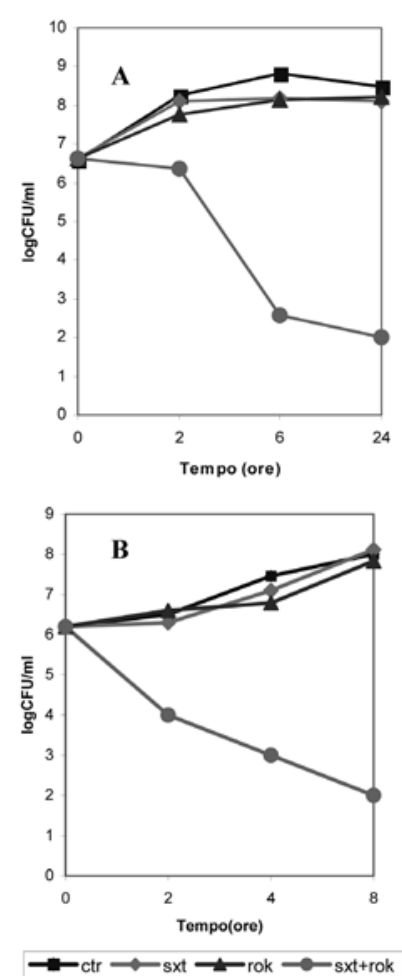

Figura III. Curve di battericidia relative a un ceppo di S. pyogenes (I 285) sensibile a SXT e con fenotipo $M$ di resistenza ai macrolidi (A) e un ceppo di S. pneumoniae $(\mathrm{CH} 40)$ resistente a SXT e con fenotipo $M$ di resistenza ai macrolidi (B)
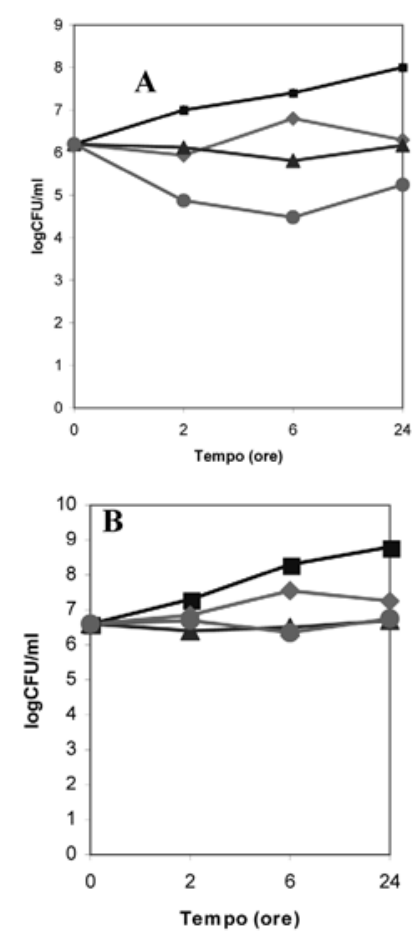

$\neg$ ctr $\longrightarrow$ sxt $\longrightarrow$ rok $\longrightarrow$ sxt+rok

Figura IV. Curve di battericidia relative a 2 ceppi di S. pyogenes con fenotipo di resistenza ai macrolidi $C M L S_{B}$, uno (GEI 2) sensibile a SXT (A) e l'altro (CH52) resistente a SXT (B) 


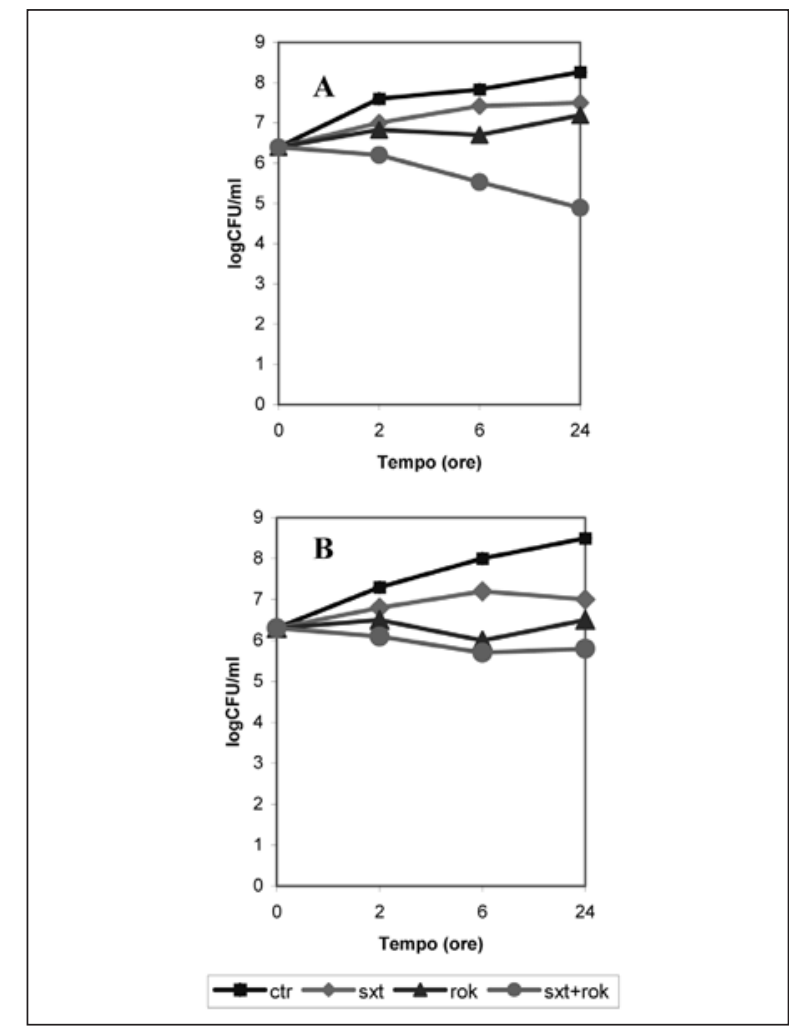

Figura V. Curve di battericidia relative a 2 ceppi di S. pyogenes con fenotipo di resistenza ai macrolidi $i M L S_{B}$, uno $(\mathrm{CH} / 4)$ sensibile a SXT e l'altro $(\mathrm{CH} 50)$ resistente a SXT

\section{BIBLIOGRAFIA}

1. Abu-Gharbieh E, Vasina V, Poluzzi E, De Ponti F. Antibacterial macrolides: a drug class with a complex pharmacological profile. Pharmacol Res 2004; 50: 211-22.

2. Chambers HF. Antimicrobial agents, protein synthesis and miscellanous antibacterial agents. In: Goodman and Gilman's the pharmacological basis of therapeutics. JG Hardman, LE Limbird, AG Gilman Eds, 10th edition. McGraw Hill, New York 2001; 1239-1271.

3. Clinical and Laboratory Standards Institute/NCCLS. Performance Standards for Antimicrobial susceptibility testing; fifteenth International Supplement. CLSI/NCCLS document M100-S15. Clinical and Laboratory Standards Institute, Wayne, Pennsylvania, 2005.

4. Giovanetti E, Montanari MP, Mingoia M, Varaldo PE. Phenotypes and genotypes of erythromycin-resistant Streptococcus pyogenes strains in Italy and heterogeneity of inducibly resistant strains. Antimicrob Agents Chemother 1999; 43: 1935-40.

5. Katz L, Ashley GW. Translation and protein synthesis: macrolides. Chem Rev 2005; 105: 499-527.

6. National Committee for Clinical Laboratory Standards. Methods for determining bactericidal activity of antimicrobial agents. Approved guideline M26-A, vol. 19. National Committee for Clinical Laboratory Standards, Wayne, Pennsylvania, 1999.

7. Retsema J, Fu W. Macrolides: structures and microbial targets. Int J Antimicrob Agents 2001; 18: S3S10.

8. Rosato A, Vicarini H, Bonnefoy A, Chantot JF, Leclercq R. A new ketolide, HMR 3004, active against streptococci inducibly resistant to erythromycin. Antimicrob Agents Chemother 1998; 42: 1392-6.

9. Seppälä H, Nissinen A, Yu Q, Huovinen P. Three different phenotypes of erythromycin-resistant Streptococcus pyogenes in Finland. J Antimicrob Chemother 1993; 32: 885-91.

10. Walsh C. Antibiotics: actions, origins, resistance. ASM Press, Washington DC, 2003.

11. Zhong P, Cao Z, Hammond R, et al. Induction of ribosome methylation in MLS-resistant Streptococcus pneumoniae by macrolides and ketolides. Microb Drug Resist 1999; 5: 183-8.

\section{Simona Roveta}

Sez. Microbiologia - DISCAT

Università degli Studi di Genova

Largo Rosanna Benzi 10 - 16132 Genova

Tel.: 0103537655 - Fax: 0103537698

E-mail: simona.roveta@unige.it 